\title{
Incidences of the Improvement of the Interactions Between MAC and Routing Protocols on MANET Performance
}

\section{Sofiane Hamrioui, Mehammed Daoui, Linda Chamek, Mustapha Lalam and Pascal Lorenz}

\author{
Houari Boumediene University, Algiers, Algeria \\ E-mail: s.hamrioui@gmail.com \\ Mouloud Mammeri University, Tizi Ouzou, Algeria \\ E-mail: \{daoui, lindachamek, lalamustapha\}@yahoo.fr \\ Haute Alsace University, Colmar, France \\ E-mail: pascal.lorenz@uha.fr
}

\begin{abstract}
In this paper, we present an improvement for the interactions between MAC and routing protocols to better energy consumption in MANET (Mobile Ad hoc Networks) and show its incidences on the performance of the network. We propose a new approach called IMREE (Improvement of the Interactions between MAC and Routing protocol for Energy Efficient) which exploits tow communication environment parameters. The first one is the number of nodes; our approach reduces the additional energy used to transmit the lost data by making the size of the backoff interval of MAC protocol adaptable to the nodes number in the network. The second parameter is the mobility of nodes; IMR-EE uses also the mobility of nodes to calculate a fairness threshold in order to guarantee the same level of the residual energy for each node in the network. We evaluate our IMR-EE solution with NS (Networks Simulator) and study its incidences on data lost and energy consumption in the network under varied network conditions such as load and mobility. The results showed that IMR-EE outperform MAC standard and allows significant energy saving and an increase in average lifetime of a mobiles nodes in the network.
\end{abstract}

Keywords: Mobile ad hoc network, Interaction between Protocols, MAC protocol, Routing protocols, IMR-EE approach, Data loss, Energy efficient, Performance evaluation. 


\section{Introduction}

The energy consumption is among the very important investigation research in MANET [1]. Many solutions have been proposed in this way and several of them use protocols from different levels of OSI model such MAC (Medium Access Control) and routing protocol [2] [3]. We exploit this policy in order to propose a new approach for better energy consumption in MANET. Our approach is IMREE (Improvement of the Interactions between MAC and Routing protocols for Energy Efficient) which is an improvement of MAC and routing protocols to their better interactions. This new approach is based essentially on the environment communication parameters especially the number of nodes in the network and their mobility. IMR-EE makes an adaptation between the number nodes in the network and the size of the backoff interval to reduce the consummation of the energy by reducing the retransmission of data lost caused by the collisions between nodes. Our solution also uses a fairness threshold of consummation energy which allows fair energy consumption between nodes. With this threshold, nodes with small value of energy will be avoid in the routing process and change statue to sleep mode in order to maintain similar power values for all the mobile nodes.

In what follows, after a short presentation of the context, we give the most significant approaches proposed for energy consumption in MANET and oriented MAC and routing levels. Then we turn to the presentation of our IMR-EE approach and it implementation in NS2. We finish our paper by study the impacts of IMR-EE on the network performance, particularly on the data loss and the energy consumption.

\section{Presentation of the Context}

A MANET (Mobile Ad hoc Network) [1] is wireless network with a high number of wireless nodes where each one communicates over the radio interface, directly or through its neighbors, without any centralized administration. Every node acts as a router for establishing the connection between each source/destination couple. The communication protocols will offer an autonomous network conceived and formed by the set of the participant nodes.

In such network, the most significant functionality is to know which node has the right to emit at a given time, where the need of protocols in order to solve this problem. These protocols must indicate the next node which will be authorized to send data on the network and they are stored in the MAC (Medium Access Control) layer [4]. Another significant functionality in MANET is routing [5], which gathers a set of procedures which start and maintain communications between two nodes. In MANET, it is necessary to create new protocols which guarantee the new needs of applications with taking into account the new 
parameters of the network (like mobility, asymmetrical links, hidden nodes, etc). These protocols can be classified according to several criteria in various families, the most used in MANET are: the link state/distance vector classification and Proactive/Reactive classification.

Among the limits to which are confronted these protocols in their functionality is how to guarantee a better energy consumption which is the objective of our present work. Many solutions was proposed in the literature and roughly classified in three families: energy control protocols, routing/MAC protocols with energy efficient and energy management protocols [2] [3].

In the following section, some suggested approaches for better energy consumption in mobile ad hoc network are given. In our work, we interested only on the approaches aiming for MAC level.

\section{Related Work}

SPAN [6] is a distributed and random algorithm for coordinators choice. Each node decides to be a coordinator or not. The transition between the two states is based on probabilities. The equity is ensured in making the node with highest energy the most probable to be a coordinator. The other criterion employed in coordinators choice is the value which a node adds to the total connectivity of the network. A node connecting more nodes will have more chances to be elected coordinator. The random concept is employed to avoid simultaneous multiple coordinators. For efficiency, these emissions are integrated in the control messages of the routing protocol (piggy-backed).

PAMAS (Power-aware Multi Access Protocol with Signaling) [7] preserves nodes power by deactivating those which do not transmit or which do not receive. It is a combination of the original protocol MACA (Chen et al., 2001), and the use of a separated channel for an activity signal. By using the activity signal, terminals are able to determine when and how long they should extinguish their radio interfaces. In this protocol, if a node does not have any packet to transmit and if one of its neighbor nodes starts transmitting, it should extinguish its radio interface. In the same way, if at least one nearby node transmits and another receives, the node should be deactivated because it cannot transmit or receive packets.

The IEEE protocol 802.11 PSM [8] proposes two modes of power management: an active mode (AM) or a power saving mode (PS). In active mode, a node is activated and can receive data at any time. In power saving mode, a packet will be delivered to a node when it is activated. The node that first transmits a beacon cancels backoff timers of nearby nodes for beacon transmission. All nodes in the network are synchronized to awake periodically. Broadcast/multicast or unicast messages for a node in power saving mode are announced via an ad hoc traffic indication message (ATIM) within a small interval called ATIM window at the beginning of the beacon interval. 
STEM (Sparse Topology and Power Management) [9] uses an independent control channel to avoid clock synchronization required by IEEE 802.11 PSM. STEM is based on asynchronous tag packets in a second control channel to awake anticipated receivers. At the end of a transmission (for example after a timeout, etc), a node deactivates its radio interface in the data channel. STEM does not provide mechanisms to indicate the state of power management of a node. Instead of that, the state of power management is only maintained in a table which is shared by all nodes taking part in data communication.

S-MAC [10] is a MAC protocol with efficient power for wireless sensors networks. S-MAC uses the listening and periodic deactivation model in order to reduce power consumption by avoiding the empty listening. However, this requires synchronization between neighboring nodes. S-MAC uses synchronization to form virtual groups of nodes on the same sleep list. This technique coordinates nodes in order to minimize additional latency. S-MAC uses channel signaling to put the nodes in 'sleep' mode when their neighboring nodes are transmitting. Channel signaling contributes to the reduction of the listening problem and avoids additional use of the channel.

F-PCM protocol [11] has used the fragmentation technique. A large DATA packet is fragmented into several small fragments and the ACK packet corresponding to each fragment is transmitted at maximum power. For each fragment transmission, maximum power for duration at the beginning of fragment transmission thus reduces collision at the sender. ACK for each fragment transmitted with maximum power will reduces collision at receiver.

A power controlled dual channel (PCDC) MAC protocol proposed in [12] allows the MAC layer to indirectly influence the routing decision at the network layer by controlling the power level of the broadcasted RREQ packets to produce power efficient routes. PCDC uses the signal strength and the direction of arrival of the overheard RTS or CTS packets to build a power-efficient network topology. PCDC enables simultaneous interference-limited transmissions to take place in the vicinity of a receiver by allowing a receiver-specific, dynamically computed interference margin.

The CDS (Connected Dominating Set) approaches [13] use vicinity or topology information to determine the set of nodes which form a CDS (connected dominating set) for the network, where any node is either a CDS member or a direct neighbor of at least one of the members. Nodes in the CDS are regarded as routing pivots and are constantly active in order to maintain the overall connectivity. Any other node can choose to put itself in sleep mode if necessary.

The GAF (Geographic Adaptive Fidelity) [14] is another technique that exploits the knowledge of nodes geographical positions to choose coordinators. Nodes geographical positions are used to divide the complete topology into fixed size zones (fixed geographical sector). The zones are created so that any two nodes in two adjacent zones can communicate. The nodes radio range, assumed fixed, dictates the size of a zone. Only one node in each zone must be awake and can be the coordinator. Thus, by exploiting the knowledge of geographical positions, 
GAF simplifies the coordinator selection procedure. Nodes in the network still commute between themselves for the work of the coordinator. The performance of GAF is biased (partial) because of the way with the zones are created and can lead to more load on some nodes than on others.

Localized Energy Aware Routing (LEAR) Protocol [15] is based on DSR but modifies the route discovery procedure for balanced energy consumption. In LEAR, a node determines whether to forward the route-request message or not depending on its residual battery power (Er). Conditional max-min battery capacity routing (CMMBCR) Protocol [16] uses the concept of a threshold to maximize the lifetime of each node and to use the battery fairly.

In [17], a new version of AODV for energy efficient is proposed to reduce energy expenditure due to overhearing. The proposed algorithm controls the level of overhearing. It reduces energy consumption without affecting quality of route information. This algorithm enables the sender to select no overhearing, unconditional overhearing or probability based overhearing for its neighbors. It is specified in the ATIM frame's sub type field and made it available to its neighbors during ATIM window. Number of overhearing nodes is controlled by probability based overhearing method.

EOLSR [18] is a variant of OLSR, where MPR selection and path calculation is determined by both a node's residual energy level and its number of neighbors. The key insight here is that sending data to a node also forces all its neighbors to consume energy in overhearing the data packet. The simulation results reported in show that combining both the new path calculation with the modified MPR selection yields the best performance. EOLSR suggests that a node's residual energy level is propagated by extending the protocol control messages, but does not discuss how accurate this information is.

In [19], the authors propose two novel mechanisms for the OLSR routing protocol, aiming to improve its energy performance in MANET. They propose a modification in the MPR selection mechanism of OLSR protocol, based on the Willingness concept, in order to prolong the network lifetime without losses of performance (in terms of throughput, end-to-end delay or overhead). Additionally, we prove that the exclusion of the energy consumption due to the overhearing can extend the lifetime of the nodes without compromising the OLSR functioning at all. A comparison of an Energy-Efficient OLSR (EE-OLSR) and the classical OLSR protocol is performed, testing some different well-known energy aware metrics such as MTPR, CMMBCR and MDR.

The previous approaches don't address the parameters of the communication environment involved in the quick energy consumption. Some works studied only the parameters related to the network traffic and focus on the MAC and routing layers separately, without giving any important for their interactions. In fact, the modeling of these parameters and their behaviors in approach based on the interactions between layers to better energy efficient will be a great contribution for MANET performance. Our work focuses precisely on these parameters in order to provide an efficient and fairness solution for energy consumption in 
MANET. In the next sections, we present our IMR-EE approach and study its incidences on MANET performance.

\section{IMR-EE (Improvement Interactions Between MAC and Routing Protocols for Energy Efficient)}

The principle of IMR-EE is to adapt the behavior of MAC and routing protocols according to two significant parameters of the communication environment which are the nodes number in the network and their mobility.

We have showed in our previous work [20] [21], that when the nodes number in the network increases, the collisions between nodes are frequent. These collisions lead to more retransmission of the data lost then to more consumption of the energy. These collisions become more frequent with a small backoff interval, because it is more probable that two nodes or more choose the same value from a larger interval than from a small one.

Note by $I$ this interval, $S_{I}$ its size, and $\operatorname{Pr}(i, x)$ is the probability that the node $i$ chooses the $x$ value from the $I$ interval. The principle is how to ensure that for any two nodes $i$ and $j$ in the network with $i !=j$, we will get:

$$
|\operatorname{Pr}(i, x)-\operatorname{Pr}(j, x)|=y \quad \text { with } y !=0
$$

For an important number of nodes in the network, and for a high probability that the formula (1) will be verified, we must have a larger $S_{I}$. To do this we will make the size of $S_{I}$ adaptable to the number of nodes in the network, then we intervene on one of the limits of this interval, and then we propose the maximum limit of $C W_{\max }$.

Note by $n$ the number of nodes in the network, and then the first part of the expression of $C W_{\max }$ will be:

$$
F(n)=\log (n)
$$

$\log ($ ) is used here because we found in [20] and [21] that the effects of the large values of the number of nodes on the number collisions respect a logarithmic function with $\mathrm{n}$ as variable.

With our solution, each node must have a local image for the variable $n$. This variable will be constantly updated whenever there is a new mobile node joins the network or leaves it. The information about $\mathrm{n}$ is broadcast as well as other information about the ad hoc network with a piggy backing technique. Many diffusion algorithms exist [22] and should be implemented with our solution to ensure the availability of the information $\mathrm{n}$ for all the nodes in the network. Upon 
receipt of $n$, all existing nodes in the network (those who are already registered and associated with MANET) will generalize this new value of $n$ on those which they have at their level. For a new node mobile in the network, upon receipt of $n$, it increments $\mathrm{n}$ by 1 , stored it locally and broadcasted to the whole network. When any node leaves the network for one reason or another, it decrements the value of $\mathrm{n}$ and diffuse it for the others nodes.

The second part of our proposed IMR-EE considers the mobility of nodes because it participates in the consumption of the energy. In fact, node mobility often leads to the breakdown of connectivity between nodes, resulting in data lost and then the transmission of these data. The frequent transmission of these data by the same node leads to the unfairness of the energy consumption. This unfairness of the energy consumption is more probable to be the cause of the break of the network and the end of the activity in the network. For this reason, our solution uses also another threshold to guarantee the same level of the residual energy for each node in the network. This threshold gets its values according to the nodes mobility and updated after each change made to this mobility.

Mobility is generally characterized by its speed and angle of movement. These two factors determine the degree of the impact of mobility on packets loss and then on the energy consumption. Consider a node $i$, in communication with another node $j$, then we note by:

$\alpha_{i, j}$ : the angle between the line $(i, j)$ and the movement direction of node $i$,

$S_{i}$ : the speed of mobile node $i$.

To consider the impact of mobility on the loss of packets is equivalent to considering the impact of its two parameters, $S_{i}$ and $\alpha_{i, j}$. For the effect of speed $S$, as in the case of number of nodes, we use a logarithmic function because for large values of speed the effect of the mobility begin to be stable, results converge. So this is expressed as follows:

$$
H\left(S_{i}\right)= \begin{cases}1 & \text { if } S_{i}=0 \text { (Without mobility) } \\ \log \left(S_{i}\right) & \text { else }\end{cases}
$$

Also, the direction of node movement determines the degree of the influence of mobility on data loss; it is given by $G\left(S_{i}, \alpha_{i, j}\right)$ like follows:

$$
G\left(S_{i}, \alpha_{i, j}\right)= \begin{cases}1 & \text { if } S_{i}=0 \quad \text { (without mobility) } \\ 1 & \text { if }-\Pi / 4<=\alpha_{i, j}<=\Pi / 4 \\ \sqrt{S_{i}} & \text { else }\end{cases}
$$

IMR-EE allows each node i to get the value of its current $S_{i}$. The easiest way to do it is to deduce it by knowing the time spent between two geographical points. There are many systems for nodes mobiles location such us GPS and power 
measurement techniques [23] [24]. With these systems, each node can know its position at any time, and then it will be able to deduce the distance travelled during an interval of time. With the distance and time we can get the speed of the mobility $S_{i}$.

Same for $\alpha_{i, j}$ which represents the angle between the direction of movement and the direction of the communication. With these location systems it is possible to determine the information about node positions node and its corresponding node and the direction of their movements. With all these information each node is able to determine the value of the angle $\alpha_{i, j}$.

From (3) and (4), we deduce that the impact of the mobility on the energy consumption will be:

$$
H\left(S_{i}\right) \wedge G\left(S_{i}, \alpha_{i, j}\right)
$$

The operator $\wedge$ (logic and) is changed by the product $*$, then we will have the finale expression of our fairness threshold $T_{i}$ for each node $i$ as follow:

$$
T_{i}=H\left(S_{i}\right) * G\left(S_{i}, \alpha_{i, j}\right)
$$

This $T_{i}$ will be used to determinate the nodes which must switch to the saving energy mode and that as follow:

If we assume that:

$E S_{i}$ is the initial energy of node $i$, which corresponds to $100 \%$, after calculating the value of $T_{i}$, the equivalent percentage is calculated, if $L M T_{i}$ is this value, then we have:

$$
\begin{aligned}
& E S_{i} \rightarrow 100 \% \\
& T_{i} \rightarrow L M T_{i} \%
\end{aligned} \text { Then } L M T_{i}=\left(T_{i} * 100\right) / E S_{i}
$$

Once $L M T_{i}$ is calculated, it is compared to the level of the remaining energy of node $i$, the following algorithm shows how $L M T_{i}$ is used to determine in which state the node $i$ must be.

We give in what follows a pseudo algorithm which shows how our new fairness of energy consumption parameter $T_{i}$ is used by the IMR-EE protocol. We assume also that:

$N$ : the number of nodes in the network;

Node $_{\mathrm{i}}$ : the node number $\mathrm{i}$;

$E N G_{\mathrm{i}}$ : the residual energy (current) of Node $_{i}$. 


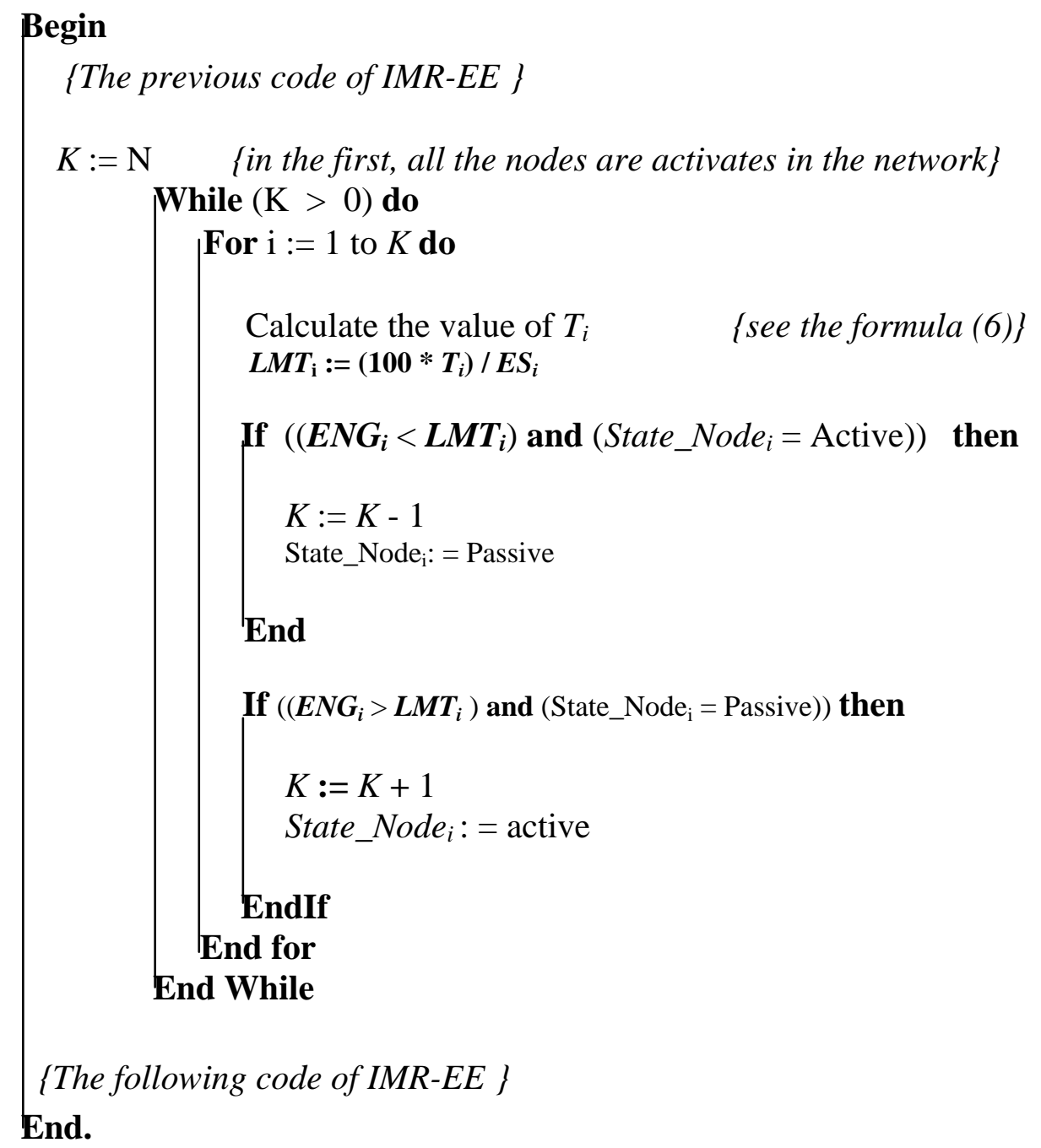

\section{Evaluation of IMR-EE}

\subsection{Simulation Environment}

The evaluation is done with NS-2 (version 2.34) [25], the MAC level uses the 802.11b model with the DCF (Distributed Coordination Function) which the values of its basic parameters are listed in the in Table 1 below.

All nodes communicate through wireless links in half-duplex with an identical bandwidth of $1 \mathrm{Mb} / \mathrm{s}$. For our simulations, the effective transmission range is of 250 meters and an interference range of 550 meters. All nodes in the area of this distance of a transmitting node will find the medium busy. Each node has a queue buffer link layer of 50 packets managed with a mode drop-tail [26]. The scheduling packet transmissions technique is the First in First out (FIFO) type. The propagation model used is the two-ray ground model [27]. 
Our simulations are done with AODV [28] as routing protocol. We study a random topology with various values of the mobility. The mobility model uses the random waypoint model [29] which is widely used in the literature. In this model the node mobility is typically random and all nodes are uniformly distributed in space simulation. The nodes move in $2200 \mathrm{~m}^{*} 600 \mathrm{~m}$ area, each one starts its movement from a random location to a random destination. Once the destination is reached, another random destination is targeted after a pause time. We used TCP NewReno [30] as transport protocol which is a reactive variant, derived and widely deployed.

Table 1: Parameters for IEEE 802.11 MAC

\begin{tabular}{|l|l|}
\hline Parameters & Values \\
\hline Preamble length (bit) & 144 \\
RTS length (bit) & 160 \\
CTS/ACK length (bit) & 112 \\
MAC header (bit) & 224 \\
IP header (bit) & 160 \\
SIFS $(\mu \mathrm{s})$ & 10 \\
DIFS $(\mu \mathrm{s})$ & 50 \\
Slot time $(\mu \mathrm{s})$ & 20 \\
Contention window & 31 \\
Retry limit & 7 \\
\hline
\end{tabular}

\subsection{Simulations results and interpretations}

\subsubsection{The data loss with the variation of the nodes number}

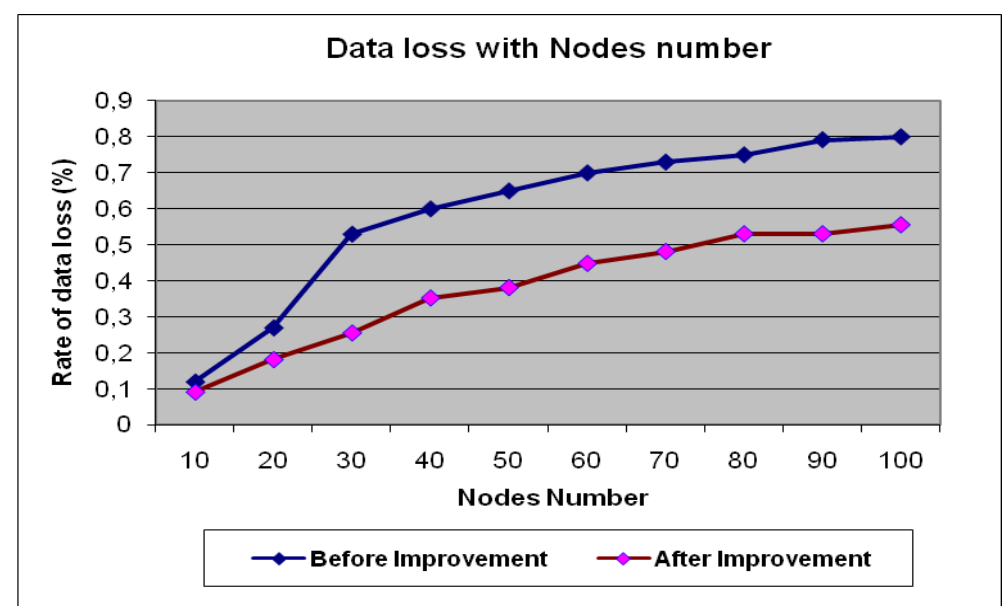

Fig. 1: Loss data with nodes number 
Fig.1. shows that before improvement, the rate of data loss increases with the increase of the nodes number more than after improvement. In fact, when the number of nodes increases in the network, the collisions between nodes becomes frequent, this makes the lost data important too. With our IMR-EE solution, we got better results with reducing considerably the rate of data loss. This improvement is because the size of the backoff interval used by our IMR-EE solution is adaptable according to the nodes number in the networks. With this adaptation, our approach avoid that the nodes chose the same probability to send or to resend their data, then the data loss is reduced compared to the case before improvement.

By exploiting the number of nodes, our approach increase considerably the performance of MAC protocol and make it more suitable to be used in MANET. In such environment, the energy consumption is important and must be reduced as more as possible and one of the ways to do it is to reduce the collisions between nodes.

\subsubsection{The data loss with the variation of nodes mobility}

For this simulation a network load is fixed to 20 sources.

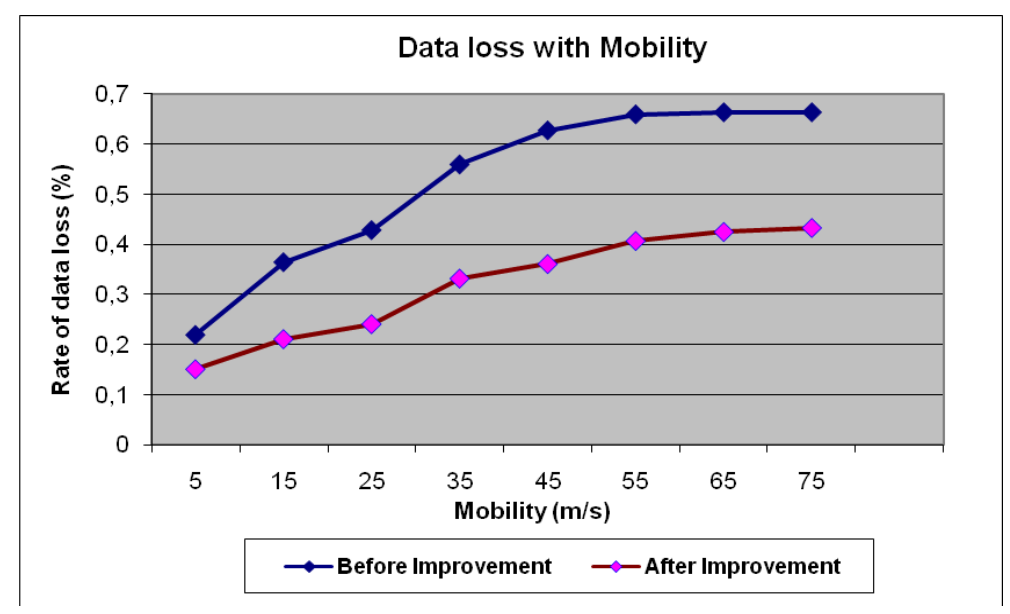

Fig. 2: Rate of data loss with mobility of nodes

For small values of nodes mobility, the network presents better stability than when these values are important. In fact, with small mobility, links failure is less frequent than in the case of a high mobility, consequently, the fraction of data loss is smaller when nodes move with low speeds, and grows with the increase of this mobility. When the loss of data is not important, the retransmission of data lost will be reduced and nodes consume less energy. Here too, our solution presents better performance; this difference in performance is because our IMR-EE uses a fairness threshold in the energy consumption updated according to evolution of 
the nodes number and their mobility in the network. With this threshold, the routing protocol used (AODV here) keeps as possible as the same values of the residual energy for each node. With this fairness in a consumption of the energy between nodes, the life of nodes will be extended which lead to the continuation in the communication as long as possible and avoid the lost data due to the life end of some nodes in the network. Then our solution, although it aims to improve the energy consumption in the network, it also reduce the lost data in the network even when the nodes are mobiles.

\subsubsection{Energy Consumption}

In this scenario, we fix the number of nodes to 50 nodes, $25 \mathrm{~m} / \mathrm{s}$, simulation duration $=1000 \mathrm{sec}$ and the initial power $=250$ joule.

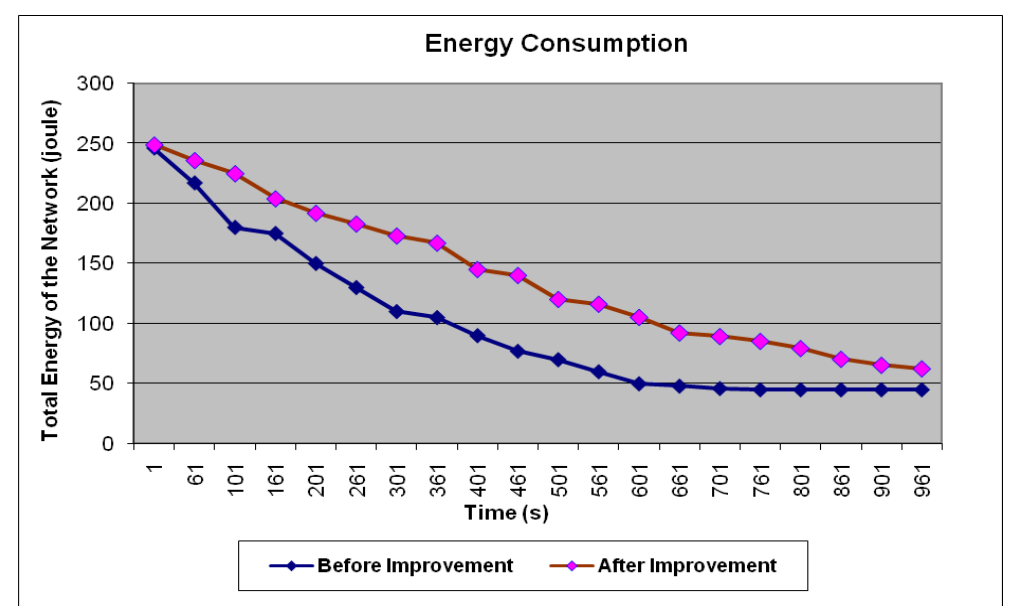

Fig. 3: Total energy of the network

Fig. 3 shows that, during the time interval $[0,600]$ sec, the total energy of the network before improvement decreases faster than after improvement. The difference in consumption during this interval is due to the intelligent management of this energy with the two improvements provided by IMR-EE particularly a fairness threshold of the energy consumption. With this threshold, the evolution of the energy will be almost same for all the nodes then their life will be extended and the network activity continues normally. This activity is fairly distributed between the nodes with the respect of the energy threshold of each one. During the interval $[600,1000]$ sec., we notice that the energy level of the network before improvement stabilizes because the activity of the network is stopped. This is because there is a loss of connectivity in the network after the total extinction of some nodes.

This extinction of the activity is the fact that the energy of nodes is used with 
unfair way, leading to depletion of the total energy of the nodes the mostly solicited in the activity of the network. This explains the failure of network connectivity, which stops the activity in the network. Indeed, when $t=600 \mathrm{sec}$, the number of working nodes in the network before improvement is 9 , and these nodes probably do not communicate because of their distance. During the same time interval, the energy in the network with our IMR-EE improvement continues to decrease, which proves that the nodes are communicating.

\subsubsection{Network's Life time}

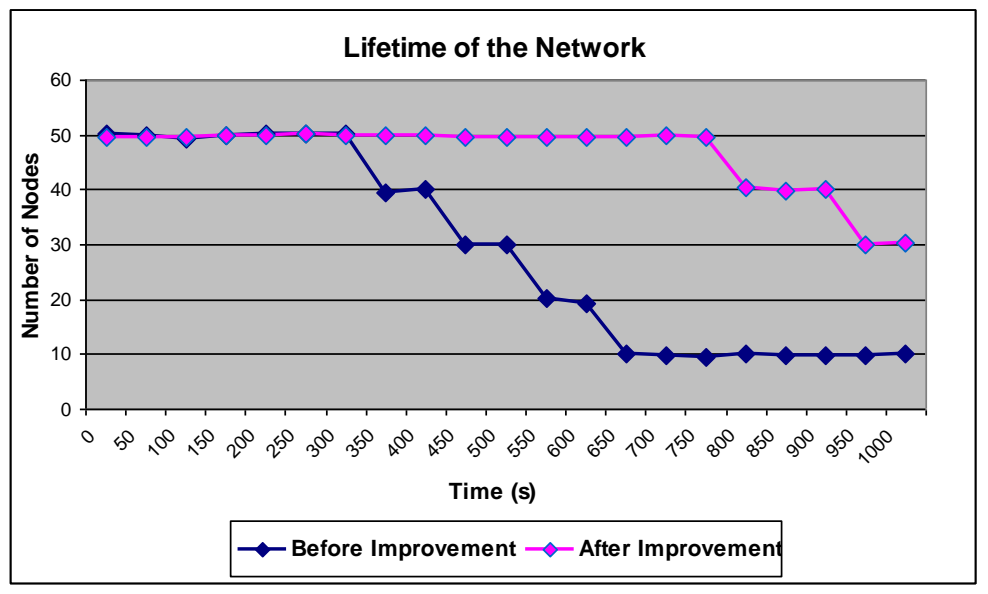

Fig. 4: Lifetime of the network

Fig. 4. shows the evolution of the lifetime of the network in the time. We see that the number of working nodes in the network before improvement decreases starting from $\mathrm{t}=250 \mathrm{sec}$ and stabilizes at $\mathrm{t}=620 \mathrm{sec}$ with only 9 nodes because of connectivity loss. On the other hand, for our proposed IMR-EE improvement, the number of working nodes in the network remains constant until $t=800 \mathrm{sec}$., then it starts to decrease rapidly.

Due to the utilization of our fairness threshold, IMR-EE favors nodes with a higher residual energy and carries out its equitable use. The intelligent management of nodes energy with our new approach allows each time to determine what nodes should switch to passive mode in order to maintain their energy level fair with the others. With this management, IMR-EE allows to extend the lifetime of nodes as long as possible. Therefore, the results of the simulations showed that our approach allows an increase in the average lifetime of nodes, and consequently allows increasing the lifetime of the whole network. 


\section{Conclusion and Future Work}

In this paper, we proposed a new approach called IMR-EE (Improvement of the Interactions between MAC and Routing protocols for Energy Efficient) which is an improvement of MAC and routing protocols for their better interactions. This new approach is based essentially on the environment communication parameters especially the number of nodes in the network and their mobility. IMR-EE makes an adaptation between the number of nodes in the network and the size of the backoff interval to reduce the consummation of the energy by reducing the retransmission of data lost caused by the collisions between nodes. Our solution also uses a fairness threshold of consummation energy which allows fair energy consumption between nodes. With this threshold, nodes with small value of energy will change statue to sleep mode in order to maintain similar power values for all the mobile nodes

After implementation and simulation of IMR-EE, we studied its incidences on a MANET performance, more particularly on data lost and energy consumption in the network. The obtained results are very conclusive and satisfactory: IMR-EE reduced data loss rates and allows a significant energy saving and an increase in average lifetime of a mobile node.

As perspectives, we will continue our work with the modeling of the maximum number of communication environment parameters. We will try to reflect as much as possible the communication environment. Our IMR-EE protocol will be compared with others solutions proposed in the same context and will be tested on a real platform; in this case, we really need to produce all the phenomena supposed exist in a real MANET.

\section{References}

[1] A. Ahmad, Wireless and Mobile Dated Networks, Wiley-Interscience Published by John Wiley \& Sons, Inc, Hoboken, New Jersey, Canada, , ISBN13 978-0-471-67075-9, ISBN-10 0-471-67075-8, 2005.

[2] L. Li, J.Y. Halpem, ' Minimum-power Mobile wireless networks revisited', in International IEEE Conference one Communications, (2001).

[3] A. Singh, H. Tiwari, A. Vajpayee, S. Prakash, , "A Survey of Energy Efficient Routing Protocols for Mobile Ad-hoc Networks', (IJCSE) International Journal on Computer Science and Engineering, Vol. 02, No. 09, 3111-3119, (2010).

[4] G. Anastasi, E. Borgia, M. Conti, E. Grego, "IEEE 802.11 Ad hoc Net works: Measurements performance", In Proceedings of the 23rd International Conference one Distributed Computing Systems Workshops (ICDCSW' 03), pages $758-763$, (2003). 
[5] P. Mohapa, S. V. Kkishnamurthy, Ad hoc Networks Technologies and Protocols, Springer Science + Business Media, Inc, eBook ISBN: 0-38722690-7, Print ISBN: 0-387-22689-3, (2005).

[6] B. Chen, K Jamieson, H. Balakrishnan, and R. Morris, "Span: Power-efficient year coordination algorithm for topology maintenance in ad hoc wireless networks", In Proc. of ACM/IEEE $7^{\text {HT }}$ Int' L Conf. one Mobile Computing and Networking (MobiCom 2001), (2001).

[7] S. Singh, C.S. Raghavendra, "Power Aware Multi-Access protocol with Signaling for ad hoc networks," ACM Computer Review Communication, vol. 28 No 3, pp. 5-26, (1998).

[8] L. Li, J.Y. Halpem, "Minimum-power Mobile wireless networks revisited," in International IEEE Conference one Communications, (2001).

[9] C. Schurgers, V. Tsiatsis, M. Srivastava, "STEM: Topology Management for Efficient Power Sensor Networks," IEEEAC, (2001).

[10] W. Ye, J. Heide mann, D. Estrin, "Power-Efficient MAC Protocol for Wireless Sensor Networks, In Proceedings of the IEEE INFOCOM , (2002).

[11] D. Kim, C.K. Toh, "F-PCM: A fragmentation-based power control MAC protocol for IEEE 802.11 mobile ad hoc networks," Wireless communication and Mobile Computing, vol. 6, n 5, 198 p., (2006).

[12] A. Muqattash, M. Krunz, Power controlled dual channel (PCDC) medium access protocol for wireless ad hoc networks, INFOCOM 2003. TwentySecond Annual Joint Conference of the IEEE Computer and Communications, 470 - 480 vol.1, (2003).

[13] M. Cardei, M.X. Cheng, X Cheng, and D.-Z Of, "Connected Domination in Ad hoc Wireless Networks," Proceedings of the International Sixth Conference one Computer Science and Informatics (CSI), (2002).

[14] Y. Xu, J. Heidemann, and D. Estrin, "Geography-informed power conservation for ad hoc routing," In the Proceedings of the 7th International Annual ACM/IEEE Conference one Mobile Computing and Networking (MobiCom' 01), Rome, Italy, (2001).

[15] K. Woo, C. Yu, D. Lee, H.Y. Youn, and B. Lee, "Localized routing algorithm for balanced energy consumption in mobile ad hoc networks', Proceedings of 9th International Symposium on Modeling, Analysis and Simulation of Computer and Telecommunication Systems, 15-18 August, Cincinnati, OH, pp.117-124, (2001).

[16] C-K. Toh, "Maximum battery life routing to support ubiquitous mobile computing in wireless ad hoc networks", IEEE Communications Magazine, Vol. 39, No. 6, pp.138-147. (2001)

[17] N. Sumathi, and A.S. Thanamani, "Evaluation of energy efficient reactive routing protocols in QoS enabled routing for MANETS", International Journal of Computer Applications, Vol. 14, No. 2, pp.10-14, (2011)

[18] S. Mahfoudh, and P. Minet, "EOLSR: an energy efficient routing protocol in wireless ad hoc sensor networks", Journal of Interconnection Networks, Vol. 9, No. 4, pp.389-408, (2008). 
[19] F. De Rango, M. Fotino, and S. Marano, "EE-OLSR: energy efficient OLSR routing protocol for mobile ad-hoc networks", IEEE Military Communications Conference, MILCOM 2008, 16-19 November, San Diego, CA, pp.1-7. (2008).

[20] S. Hamrioui, M. Lalam, "Incidence of the Improvement of the Transport MAC Protocols Interactions on MANET Performance," 8th Annual International Conference on New Technologies of Distributed Systems (NOTERE 2008). Lyon, (2008).

[21] S. Hamrioui, M. Lalam, "Incidences of the Improvement of the MACTransport and MAC-Routing Interactions on MANET Performance," International Conference on Next Generation Networks and Services, MARRAKESH, (2010).

[22] M. Lehsaini, "Diffusion et couverture basées sur le clustering dans les réseaux de capteurs : application à la domotique," Thèse de Doctorat Spécialité Informatique, Université de Franche-Comté, (2009).

[23] D.K. Elliott, Understanding GPS: Principles and Applications, Second Edition. Artech House Publishers, (2005).

[24] L. Doherty, K.S.J. Pister, L. El Ghaoui, "Convex position estimation in Wireless sensor networks," In Proc. of the IEEE INFOCOM, Alaska, vol.3, pp.1655-1663, (2001).

[25] The Network Simulator NS-2, http://www.isi.edu/nsnam/ns/

[26] S. Floyd, V. Jacobson, "Random Early Detection Gateways for Congestion Avoidance," IEEE/ACM Transactions on Networking, Vol. 1, pp. 397-413, (1993).

[27] K. Bullington, "Radio Propagation Fundamentals," The Bell System Technical Journal, Vol. 36, No.3. 593-625, (1957).

[28] C. E. Perkins, E. M. Royer, S. R. Das, Ad Hoc On-Demand DistanceVector (AODV) Routing, IETF Internet draft (draft-ietf-manet-aodv-o6.txt).

[29] E. Hyytiä, J. Virtamo, Randomwaypoint model in n-dimensional space, Operations Research Letters 33, $567-571$, (2005).

[30] S. Floyd, T. Henderson, New Reno Modification to TCP's Fast Recovery, RFC 2582, (1999). 